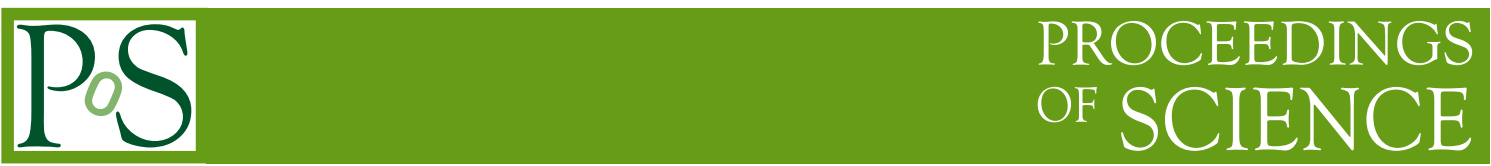

\title{
Heavy-quark production in deep-inelastic scattering
}

\section{Sergey Alekhin ${ }^{* \dagger}$}

DESY, Platanenallee 6, D-15738 Zeuthen, Germany

Institute for High Energy Physics, 142281 Protvino, Moscow region, Russia

E-mail: sergey.alekhinedesy.de

\section{Johannes Blümlein}

DESY, Platanenallee 6, D-15738 Zeuthen, Germany

E-mail: johannes.bluemlein@desy.de

\section{Sven-Olaf Moch}

DESY, Platanenallee 6, D-15738 Zeuthen, Germany

II. Institut für Theoretische Physik, Universität Hamburg Luruper Chaussee 149, D-22761

Hamburg, Germany

E-mail: sven-olaf.moch@desy.de

We report recent experimental and theoretical progress concerning the heavy-quark electroproduction in the context of the ABM11 parton distribution function (PDF) fit. In the updated ABM11 analysis, including the recent combined HERA charm data, the $\overline{\text { MS}}$-values of the $c$-quark mass $m_{c}\left(m_{c}\right)=1.24 \pm 0.03(\exp )_{-0.02}^{+0.03}(\text { scale })_{-0.07}^{+0.00}($ th $)$ and $m_{c}\left(m_{c}\right)=1.15 \pm$ $0.04(\exp )_{-0.00}^{+0.04}$ (scale) are determined at NNLO and NLO, respectively. The values of $m_{c}$ obtained are compared to other determinations including the ones based on the various variable-flavornumber (VFN) scheme prescriptions. The VFN scheme uncertainties related to the matching of the 4(5)-flavor PDFs with the 3(4)-flavor ones are discussed.

XXI International Workshop on Deep-Inelastic Scattering and Related Subject-DIS2013,

\footnotetext{
*Speaker.

$\dagger$ This work has been supported in part by Helmholtz Gemeinschaft under contract VH-HA-101 (Alliance Physics at the Terascale), DFG Sonderforschungsbereich/Transregio 9 and by the European Commission through contract PITNGA-2010-264564 (LHCPhenoNet)
} 
The $c$ - and $b$-quarks provide an important experimental and phenomenological tool to study the nucleon structure. Experimental separation of the heavy quarks in the final state is facilitated due to their relatively large masses. On the other hand, since the masses $m_{c, b} \gg \Lambda_{Q C D}$, with $\Lambda_{Q C D}$ stands for the QCD scale, the Wilson coefficients for heavy-quark production can be calculated within perturbative QCD. The study of heavy-quark production in the deep-inelastic scattering (DIS) process has been started in the fixed-target experiments. However, only at the energies available at HERA it gives a substantial contribution to the inclusive structure functions (SFs). Through the photon-gluon fusion mechanism the semi-inclusive SFs of the $c$-and $b$-quark DIS production are directly connected to the gluon distribution. Therefore they are customary employed in the parton-distribution function (PDF) analyses as an additional constraint on the small- $x$ behavior of the gluon distribution. The main theoretical difficulty arising in this context is related to the emergence of two hard scales, given by the quark mass and the DIS momentum transfer $Q^{2}$. At $Q^{2} \gg m_{c, b}^{2}$ power corrections of $O\left(m_{Q}^{2} / Q^{2}\right)$ may be neglected and the massive Wilson coefficients can be presented as a convolution of the massless coefficients with the massive operator matrix elements (OMEs) [1,2]. This approach serves a basis of the variable-flavor-number (VFN) scheme trying to overcome the difficulties of the full massive calculations. However, the asymptotic regime poorly overlaps with the kinematics of the present data at HERA, which abundantly populate the low- $Q^{2}$ region. In contrast, the fixed-flavor-number (FFN) scheme provides an accurate treatment of the mass effects at threshold. Moreover, this scheme has demonstrated very good agreement with the existing DIS data up to the largest values of $Q^{2}$ [4]. In the following we describe the impact of the new charm-production data on the ABM PDF fit [4] related to the recent theoretical progress in the FFN scheme calculations. We report the value of $m_{c}$ extracted from the DIS data alongside with the analysis of its uncertainty and discuss additional uncertainties on $m_{c}\left(m_{c}\right)$ and strong coupling constant $\alpha_{s}$ emerging in the VFN scheme.

The recent version of the ABM PDF fit [4] is based on the running-mass definition of the massive Wilson coefficients [5] with the values of $m_{c, b}$ fixed at the PDG values [6]. However, $m_{c}$ can be also determined from the $\mathrm{H} 1$ data on charm production [7] and the constraint on $m_{c}$ coming from the combined HERA charm data [8] turns out to be even more substantial. Using advantages of that experimental input we perform a variant of the ABM PDF fit with the combined HERA data added and the value of $m_{c}$ fitted simultaneously with the value of $\alpha_{s}$ and the PDF parameters [9]. A model of main massive Wilson coefficients employed in this fit has been derived in Ref. [10] as a combination of the threshold resummation results [11] with the high-energy asymptotics of the DIS structure functions [12]. These two regimes are matched using the available Mellin moments of the NNLO massive OMEs and functions [13,14]. Furthermore, the calculations are performed within the running-mass definition providing improved perturbative convergence of the result [5]. To quantify the uncertainty in the approximate NNLO coefficients obtained in this way two options of these coefficients, A and B, are provided in Ref. [10]. In our analysis we employ a linear combination of these options with an interpolation parameter $d_{N}$ fitted simultaneously with the other fit parameters. The value of $d_{N}=-0.1$ found corresponds to the coefficient shape close to option A. The option B is disfavored by the HERA charm data [8], cf. Fig. 1, with $\chi^{2} / N D P=$ $115 / 52$ obtained in the variant of the fit with this shape, where NDP stands for the number of data points. Therefore we quantify uncertainties due to the massive NNLO coefficients by the difference between the results obtained with the value of $d_{N}=-0.1$ preferred by the data and 


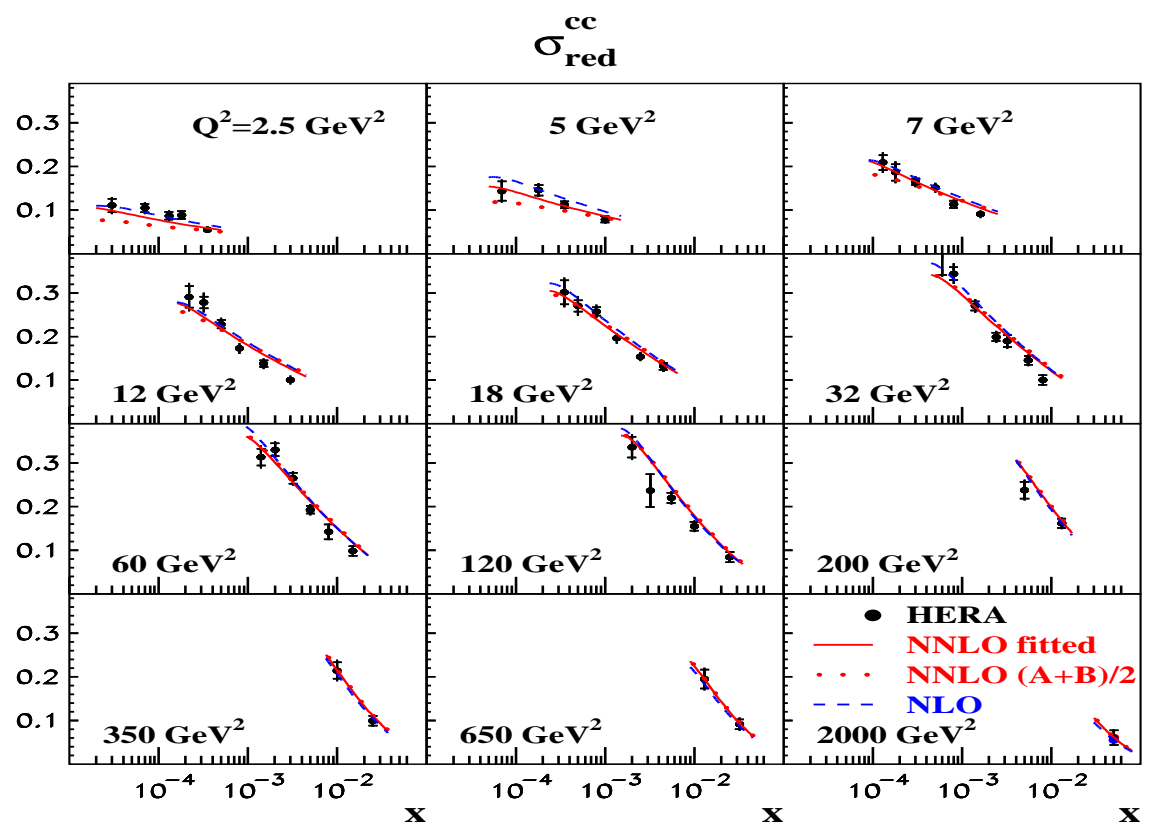

Figure 1: The combined HERA data on the open charm production [8] versus $x$ at different values of $Q^{2}$ in comparison with the analysis of [9] at NLO (dashed line) and NNLO (solid line) together with a fit variant based on the option $(\mathrm{A}+\mathrm{B}) / 2$ of the NNLO Wilson coefficients of Ref. [10] (dotted line); from Ref. [9].

$d_{N}=0.5$, corresponding to the average of the options A and B.

The PDFs obtained in this version of the ABM fit including the HERA charm data are compared with those of ABM11 in Fig. 2. The change in the sea quark distribution is moderate and the change in the valence region is even smaller. At the same time the gluon distribution changes by $1 \sigma$ in places both due to impact of the new experimental and the theoretical improvements in the heavy-quark treatment. The $\overline{\mathrm{MS}}$-values of the $c$-quark mass obtained in our analysis are

$$
\begin{array}{ll}
m_{c}\left(m_{c}\right)=1.15 \pm 0.04(\exp )_{-0.00}^{+0.04}(\text { scale }) & \text { NLO } \\
m_{c}\left(m_{c}\right)=1.24 \pm 0.03(\exp )_{-0.02}^{+0.03}(\text { scale })_{-0.07}^{+0.00}(\mathrm{th}), & \text { NNLO }_{\text {approx }}
\end{array}
$$

at NLO and NNLO, respectively. The NLO value of $m_{c}\left(m_{c}\right)=1.26 \pm 0.05$ (exp) GeV extracted form the HERA data only [8] is somewhat bigger than ours in Eq. (1). The difference between these two determinations was found to appear mainly due to the selection of the data employed in the analysis, cf. [9] for details. The theoretical errors, Eq. $(1,2)$, emerge due to the factorization scale variation by a factor of $1 / 2$ and 2 around the nominal value of $\sqrt{Q^{2}+\kappa m_{c}^{2}}$ and due to the NNLO coefficient shape uncertainty ${ }^{1}$. The NNLO central value is comparable with the one obtained from the $e^{+} e^{-}$data and the total error is competitive with the world average [6].

In comparison to the FFN scheme the VFN scheme brings in two additional uncertainty sources. The first is related to modeling of the low- $Q^{2}$ region, which is necessary to provide a reasonable behavior of the VFN scheme in the kinematic region of the present DIS data. This uncertainty was in particular quantified by the extraction of $m_{c}$ within various prescriptions of

\footnotetext{
${ }^{1}$ The factor of $\kappa$ is 4 and 1 for the neutral- and charged-current cases, respectively.
} 


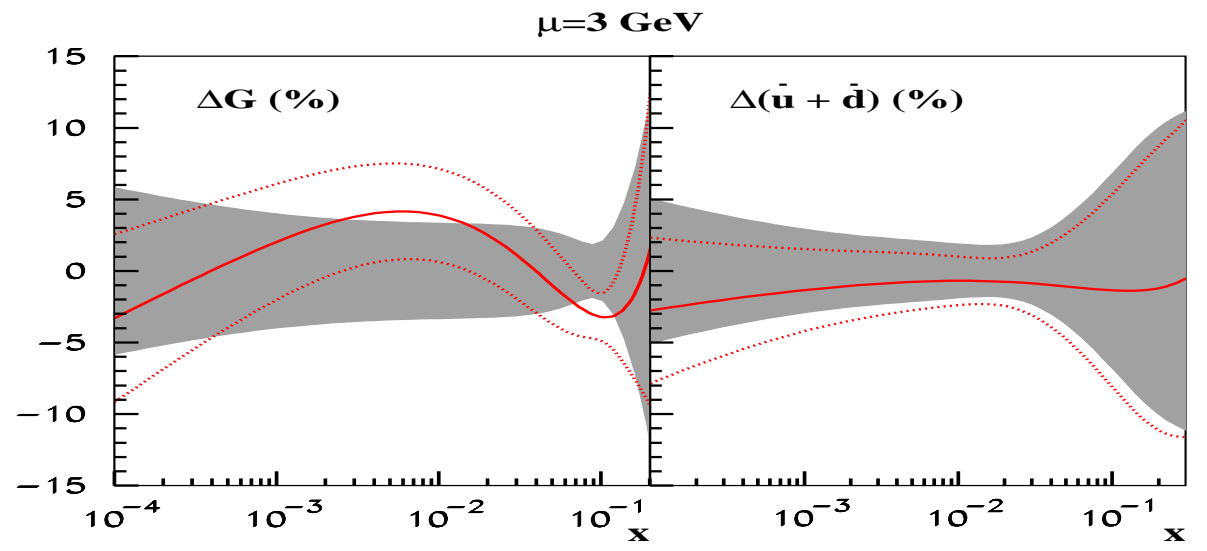

Figure 2: The relative change in the NNLO gluon (left) and non-strange sea (right) distributions obtained in the present analysis with respect to the ABM11 PDFs (solid lines). The relative uncertainties in the PDFs are displayed for comparison (shaded area: ABM11, dotted lines: present analysis).

the VFN scheme, including ACOT-full, S-ACOT- $\chi$, RT-standard, and RT-optimized prescriptions. While the quality of the data obtained with different prescriptions is similar, the value of $m_{c}$ preferred by the data differs by $\pm 200 \mathrm{MeV}$ [8]. This estimate is comparable with the uncertainty in $m_{c}$ due to variation of the S-ACOT- $\chi$ prescription parameters [15]. The second source of uncertainty is related to the generation of the 4(5)-flavor PDFs. They are commonly matched with the 3(4)-flavor ones at the scale of $\mu_{0}=m_{c}\left(m_{b}\right)^{2}$. This is an arbitrary choice of course and the variation of the

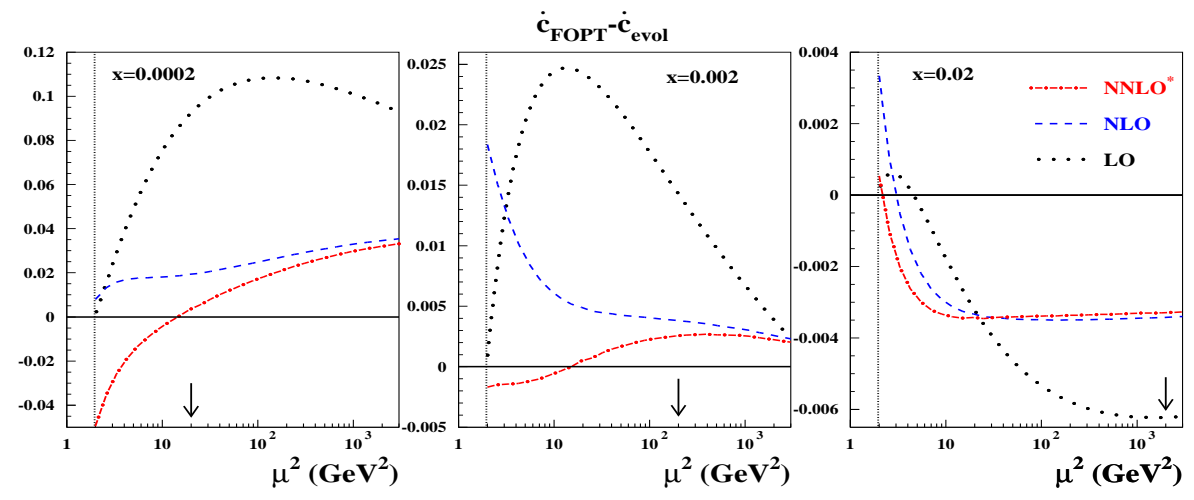

Figure 3: The difference between the $c$-quark PDFs derivatives $\dot{c}\left(x, \mu^{2}\right) \equiv \frac{d c\left(x, \mu^{2}\right)}{d \ln \mu^{2}}$ calculated with the FOPT matching condition and with the massless 4-flavor evolution starting at the matching point $\mu_{0}=m_{c}=1.4 \mathrm{GeV}$ versus the factorization scale $\mu^{2}$ at different values of $x$ in the LO, NLO, and NNLO* approximations. The arrows display the upper margin of the HERA collider kinematics with the collision c.m.s. energy squared $s=10^{5} \mathrm{GeV}^{2}$ and the vertical lines correspond to the matching point position $\mu_{0}$.

matching point $\mu_{0}$ in a wide range is allowed in principle. Further, the 4(5)-flavor PDF obtained in this way are evolved starting from the scale $\mu_{0}$ using massless evolution kernels. In the NNLO case this cannot be performed consistently since the NNLO OMEs are not yet fully known ${ }^{3}$. In

\footnotetext{
${ }^{2}$ Note, at the scale of $m_{b}$ the charm mass effects cannot be fully neglected.

${ }^{3}$ For progress in this field, cf. [16].
} 
practice, the the NNLO evolution is commonly combined with the NLO matching at $\mu_{0}$ arriving at an approximation called $\mathrm{NNLO}^{*}$ in the following. The theoretical uncertainties in the latter are illustrated by comparison of the $c$-quark distributions $c\left(x, \mu^{2}\right)$ generated at $\mathrm{NNLO}^{*}$ to the NLO ones, which are generated using the NLO matching in combination with the NLO evolution. We consider the derivative of $c\left(x, \mu^{2}\right)$ w.r.t. the factorization scale $\mu^{2}$ and take the difference of this derivative with the one calculated in fixed-order-perturbative theory (FOPT) employing the massive OMEs to produce $c\left(x, \mu^{2}\right)$ at all values of $\mu^{2}$. This representation allows to check the impact of the $\ln \mu^{2}$-resummation manifesting in the PDF evolution at large $\mu^{2}$. This resummation reproduces the higher-order correction effects in part. Therefore the difference between the FOPT and evolved PDFs vanishes with perturbative order. At NLO and NNLO* the resummation effects are numerically significant at $x \lesssim 0.0001$ and at $\mu^{2}$ outside of the HERA kinematics only, cf. Fig. 3 . In particular this signals that the FFN scheme can be reliably used in the NNLO analysis of the HERA data. At the same time the uncertainty in the NNLO* approximation of the VFN scheme is localized at small $\mu^{2}$ well covered by the HERA data. The impact of this uncertainty combined with the variation of the matching point $\mu_{0}^{2}$ within the range of $1.2 \div 1.5 \mathrm{GeV}$ on $\alpha_{s}\left(M_{Z}\right)$ is estimated as \pm 0.001 for the VFN variant of the ABM11 fit. In combination with the uncertainty due to the low- $Q^{2}$ modeling this makes the VFN scheme uncompetitive with the FFN one in the precision determination of $\alpha_{s}$.

\section{References}

[1] M. Buza, Y. Matiounine, J. Smith and W. L. van Neerven, Eur. Phys. J. C 1, 301 (1998).

[2] M. A. G. Aivazis, J. C. Collins, F. I. Olness and W. -K. Tung, Phys. Rev. D 50, 3102 (1994).

[3] S. Alekhin, J. Blümlein, S. Klein and S. Moch, Phys. Rev. D 81, 014032 (2010).

[4] S. Alekhin, J. Blümlein and S. Moch, Phys. Rev. D 86, 054009 (2012).

[5] S. Alekhin and S. Moch, Phys. Lett. B 699, 345 (2011).

[6] J. Beringer et al. [Particle Data Group Collaboration], Phys. Rev. D 86, 010001 (2012).

[7] S. Alekhin, K. Daum, K. Lipka and S. Moch, Phys. Lett. B 718, 550 (2012).

[8] H. Abramowicz et al. [H1 and ZEUS Collaborations], Eur. Phys. J. C 73, 2311 (2013).

[9] S. Alekhin et al., Phys. Lett. B 720, 172 (2013).

[10] H. Kawamura, N. A. Lo Presti, S. Moch and A. Vogt, Nucl. Phys. B 864, 399 (2012).

[11] N. A. Lo Presti, H. Kawamura, A. Vogt and S. Moch, AIP Conf. Proc. 1388, 484 (2011).

[12] S. Catani, M. Ciafaloni and F. Hautmann, Nucl. Phys. B 366, 135 (1991).

[13] I. Bierenbaum, J. Blümlein, S. Klein and C. Schneider, Nucl. Phys. B 803m 1 (2008).

[14] I. Bierenbaum, J. Blümlein and S. Klein, Nucl. Phys. B 820, 417 (2009).

[15] J. Gao, M. Guzzi and P. M. Nadolsky, arXiv:1304.3494 [hep-ph].

[16] J. Ablinger et al., PoS LL 2012, 033 (2012); J. Ablinger et al., Nucl. Phys. B 864,42 (2012); J. Ablinger et al., Nucl. Phys. B 844,26 (2011);

J. Blümlein, A. Hasselhuhn, S. Klein and C. Schneider, Nucl. Phys. B 866, 196 (2013). 